\title{
Black hole growth by accretion
}

\author{
S. Mathur and D. Grupe
}

\author{
Astronomy Department, The Ohio State University, 140 West 18th Avenue, Columbus, OH 43210, USA \\ e-mail: smita@astronomy.ohio-state.edu
}

Received 22 July 2004 / Accepted 9 November 2004

\begin{abstract}
We show that black holes grow substantially by accretion at close to Eddington rates. Using a complete sample of soft X-ray selected AGNs, Grupe \& Mathur (2004, ApJ, 606, L41) have shown that narrow line Seyfert 1 galaxies, as a class, lie below the $M_{\mathrm{BH}}-\sigma$ relation of normal galaxies. Some NLS1s, however, lie on or close to the $M_{\mathrm{BH}}-\sigma$ relation. Here we show that not all NLS1s accrete at close to Eddington rates: those with low $L / L_{\text {Eddington }}$ are close to the $M_{\mathrm{BH}}-\sigma$ relation, and those with high $L / L_{\text {Eddington }}$ are far from it. With various tests in this paper, we argue that black holes grow in mass substantially in their high-accretion phase and approach the $M_{\mathrm{BH}}-\sigma$ relation over time. The mass growth in a low accretion phase, as in BLS1s and also in some NLS1s, appears to be insignificant. Any theoretical model attempting to explain the $M_{\mathrm{BH}}-\sigma$ relation needs to explain the above observations.
\end{abstract}

Key words. black hole physics - galaxies: active - galaxies: evolution - X-rays: galaxies

\section{Introduction}

The observation of a tight correlation between the velocity dispersion $\sigma$ of the bulge of a galaxy and the mass of its nuclear black hole $M_{\mathrm{BH}}$ was a surprising discovery over the last few years (Gebhardt et al. 2000a; Ferrarese \& Merritt 2000; Merritt \& Ferrarese 2001; see also Kormendy \& Richstone 1995, and references there in). Even more surprisingly, the above relation for normal galaxies was also found to extend to active galaxies (Gebhardt et al. 2000b; Ferrarese et al. 2001; Haering \& Rix 2004). Moreover, dead black holes were found in the nuclei of all the observed nearby galaxies (e.g. Ho 1999). This was an important result because it implies that nuclear activity was perhaps a part of the life of every galaxy and that the quasar phenomenon is not just a spectacular but cosmologically uninteresting event.

Many theoretical models attempt to provide an explanation for the $M_{\mathrm{BH}}$ and bulge relations. To name a few, Haehnelt et al. (1998) discussed the formation and evolution of galaxies and their nuclear black holes in the framework of the hierarchical dark-matter cosmology. They found that the black hole mass is proportional to $M_{\text {halo }}^{5 / 3}$. In the model of Wang \& Biermann (1998), nuclear starbursts and AGNs co-exist, compete for gas supply, which ultimately constrains the blackhole-mass-to-bulge-mass ratio. Accretion plays a critical role in the model by Wang et al. (2000). These early models, however, used the early observational results of Magorrian et al. (1998) in which black hole masses were overestimated, and so resulted in conclusions which were later revised. For example, in the Wang et al. model, quasars accrete at close to Eddington ratio and so have high $\mathrm{BH}$-mass to bulge ratio and Seyferts accrete at lower rate and have lower BH-mass to bulge-mass ratio. New observational results show that this is not the case (Gebhardt et al. 2000b; Ferrarese et al. 2001). Similarly, Haehnelt et al. (1998) had to invoke black hole growth in the obscured phase to account for the large, but erroneous, local black hole mass density compared to the integrated quasar light. Later models focused on the tight $M_{\mathrm{BH}}-\sigma$ relation. For example, in the model of Haehnelt \& Kauffmann (2000), supermassive black holes form and grow during major mergers as they move along the observed $M_{\mathrm{BH}}-\sigma$ relation. On the other hand, mergers are ignored in the model by $\mathrm{Yu}$ $\& \mathrm{Lu}$ (2004). Outflows from AGNs were invoked to limit the black hole growth in a recent model by King (2003). A model by Miralda-Escudé \& Kollmeier (2003) explicitly couples accretion with the stellar system around it. They reproduce the observed $M_{\mathrm{BH}}-\sigma$ relation as the final relation at the end of the accretion process.

To understand the origin of the $M_{\mathrm{BH}}-\sigma$ relation, and to discriminate among the models, it is of interest to follow the tracks of AGNs in the $M_{\mathrm{BH}}-\sigma$ plane. Mathur et al. (2001) suggested that the narrow line Seyfert 1 galaxies (NLS1s), a subclass of Seyfert galaxies believed to be accreting at a high Eddington rate, do not follow the $M_{\mathrm{BH}}-\sigma$ relation. (NLS1s are defined as Seyfert galaxies with full width at half maximum of $\mathrm{H} \beta$ lines of less than $2000 \mathrm{~km} \mathrm{~s}^{-1}$ (Osterbrock \& Pogge 1985).) This result was later confirmed by Wandel (2002) and Bian \& Zhao (2004). Using a complete sample of soft X-ray selected AGNs, Grupe \& Mathur (2004, Paper I hereafter) determined the black hole mass-bulge velocity dispersion relation for 43 broad line Seyfert 1s and 32 narrow line Seyfert 1s. In all the three papers listed above, the authors use luminosity and $\operatorname{FWHM}(\mathrm{H} \beta)$ as 
surrogates for black hole mass, and FWHM([OIII]) as a surrogate for the bulge velocity dispersion. Grupe \& Mathur (2004) found that NLS1s lie below the $M_{\mathrm{BH}^{-}}-\sigma$ relation of BLS1s, confirming the Mathur et al. (2001) result. The statistical result was robust and not due to any systematic measurement error. As noted by Grupe \& Mathur (2004), this result has important consequences for our understanding of black hole formation and growth: black holes grow by accretion in well formed bulges, possibly after a major merger. As they grow, they get closer to the $M_{\mathrm{BH}}-\sigma$ relation for normal galaxies. The accretion is highest in the beginning and dwindles as the time goes by. While a theoretical model to explain all the observations has yet to come, the above result makes it possible to rule out a class of models: e.g. the above result does not support theories of the $M_{\mathrm{BH}}-\sigma$ relation in which the black hole mass is a constant fraction of the bulge mass/ velocity dispersion at all times in the life of a black hole, or theories in which bulge growth is controlled by AGN feedback. A broad consistency is found with the model of Miralda-Escudé \& Kollmeier (2004).

At a first glance, the above result is at odds with the observation that some NLS1s, at the low end of the observed range of velocity dispersion, lie on or close to the $M_{\mathrm{BH}}-\sigma$ relation (Mathur et al. 2001; Ferrarese et al. 2001; Bian \& Zhao 2004; and Grupe \& Mathur 2004). As mentioned above, the Grupe \& Mathur (2004) statistical result is robust, in that NLS1s as a class do lie below the $M_{\mathrm{BH}}-\sigma$ relation of normal galaxies (the Kolmogorov-Smirnov (K-S) probability of NLS1s and BLS1s being drawn from the same population is $P<0.001)$. However, the observation of some NLS1s on or close to the relation affects the interpretation of the result. If we are to interpret the observations in terms of black hole growth by the highly accreting NLS1s, why have some NLS1s already reached their "final" mass? In this paper we propose a solution to this apparent contradiction.

\section{The hypothesis}

The first hint towards the resolution of the above conflict came from the observation of Williams, Mathur \& Pogge (2004). In Chandra observations of 17 NLS1, they find a correlation between the soft X-ray power-law slope $\alpha$ and the $1 \mathrm{keV}$ luminosity (see also Grupe 2004). It has been known for many years that not all NLS1s have steep soft X-ray spectra (e.g. Boller et al. 1996; Grupe et al. 1998, 2004). The results of Williams et al. (2004) and Grupe (2004) have shown that a significant fraction of NLS1s have flat X-ray spectra and those with flatter spectra are preferentially lower luminosity objects. They have also shown that in most of them absorption is not the cause of the observed flatness of X-ray spectra.

The paradigm that NLS1s are highly accreting AGNs came from the work of Pounds et al. (1995). Theoretical models of accretion disk plus corona also confirmed that a high accretion rate relative to Eddington $(\dot{m})$ leads to steep soft X-ray spectra while low $\dot{\mathrm{m}}$ accretion results in flatter spectra (Kuraszkiewicz et al. 2000). The soft X-ray power-law slope was found to correlate strongly with $L / L_{\text {Eddington }}$ in Williams et al. (2004) and in Grupe (2004). The relatively flatter spectra in some NLS1s suggest that these objects are accreting at a substantially

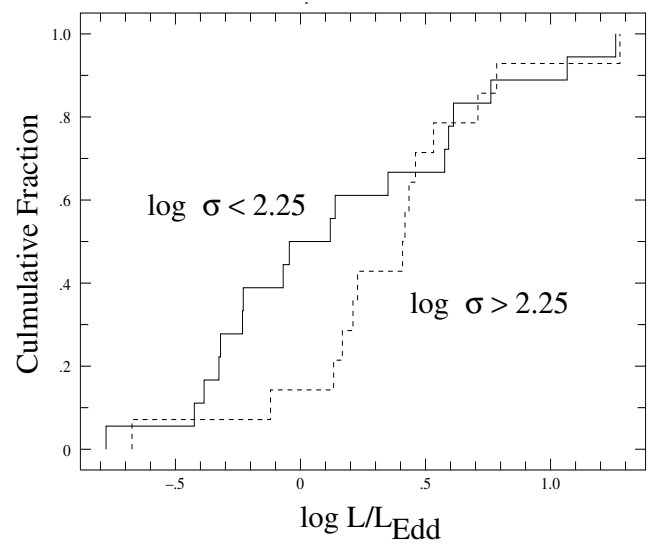

Fig. 1. Cumulative fraction for a K-S test of $L / L_{\text {Eddington }}$ for NLS1 with $\log \sigma_{[\mathrm{OIII}]}<2.25$ (solid line) and $\log \sigma_{\text {[OIII] }}>2.25$ (dashed line). The two distributions are clearly different. The NLS1s with high $L / L_{\text {Eddington }}$ occupy a distinct region on the $M_{\mathrm{BH}}-\sigma$ plane.

sub-Eddington rate, compared to the NLS1s with steep X-ray spectra. In the framework of the black hole growth scenario of Mathur et al. (2001) and Grupe \& Mathur (2004), these objects may then be the ones close to the $M_{\mathrm{BH}}-\sigma$ relation, as they would have already gone through their high m state and their black holes have accumulated most their mass. In the following section we test this hypothesis.

\section{Tests}

If the above resolution to the black hole growth scenario is correct, then we should find that the NLS1s close to the $M_{\mathrm{BH}}-\sigma$ relation have low $L / L_{\text {Eddington }}$ compared to those lying below the $M_{\mathrm{BH}}-\sigma$ relation. To test this prediction, we divided our NLS1 sample from Grupe \& Mathur (2004) in two parts, with low and high values of $\sigma$ with a boundary at $\log \sigma_{\text {[OIII] }}=2.25$. The choice of the boundary came from the visual inspection of Fig. 1 of Grupe \& Mathur (2004), where it was found that the NLS1s with $\log \sigma_{\text {[OIII] }}$ below this value tended to be much closer to the $M_{\mathrm{BH}}-\sigma$ relation. Figure 1 compares the distribution of $L / L_{\text {Eddington }}$ for the two samples. The values of $L / L_{\text {Eddington }}$ are given in Grupe et al. 2004 and those of $\sigma_{\mathrm{O}[\mathrm{III}]}$ are as in Grupe \& Mathur (2004, their Fig. 4). The K-S cumulative distribution for the two samples is significantly different, with the formal K-S test probability of being drawn from the same population $P=0.1$. This result is statistical in nature. The error in values of $L / L_{\text {Eddington }}$ for each object, as determined in Grupe et al. (2004), assuming a bolometric correction factor, may be a factor of several. The point to note here is the difference in the two populations with low and high $\sigma$ which correspond to objects close to and away from the $M_{\mathrm{BH}}-\sigma$ relation respectively. Figure 1 thus shows that the objects closer to the $M_{\mathrm{BH}}-\sigma$ relation have lower $L / L_{\text {Eddington }}$ and those lying below the relation have statistically higher $L / L_{\text {Eddington }}$.

One has to be cautious interpreting the above result, because one may obtain high values of $L / L_{\text {Eddington }}$ if black hole masses are underestimated. We have emphasized in Paper I that this is not the case; the $\mathrm{BH}$ masses in our sample are unlikely to be systematically underestimated because the 


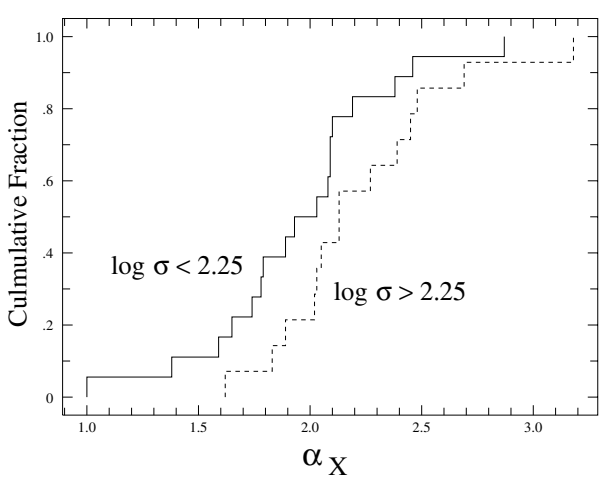

Fig. 2. Same as Fig. 1, but for the soft X-ray spectral index $\alpha$. Again, the two distributions are found to be statistically different. Objects with high $L / L_{\text {Eddington }}$ also have steep spectra and are the ones lying below the $M_{\mathrm{BH}}-\sigma$ relation of normal galaxies.

relationship between $\mathrm{H} \beta$ FWHM and the broad line region radius is well calibrated and extends to NLS1s as well. Moreover, the value of $\sigma$ plays no role in $\mathrm{BH}$ mass estimates, so even if the $\mathrm{BH}$ masses were underestimated, they would be so for all NLS1s, irrespective of $\sigma$ and not for high $\sigma$ objects only. Secondly, BH mass estimates using two completely different methods give the same result: in Mathur et al. (2001), $M_{\mathrm{BH}}$ was determined by fitting accretion disk models to SEDs and in Czerny et al. (2001) power-spectrum analysis was used. Nonetheless, another test of the above hypothesis may be a comparison of the X-ray power-law slopes of the two populations of high and low $\sigma$. If our hypothesis is correct, and if steep and flat X-ray spectra result in NLS1s with high and low $L / L_{\text {Eddington }}$ respectively, then we should find that the NLS1s with low values of $\sigma$ i.e. those close to the $M_{\mathrm{BH}}-\sigma$ relation have flatter $\alpha$ (and lower m) compared to NLS1s with high values of $\sigma$. In Fig. 2 we plot the K-S cumulative distribution of $\alpha$ for the two populations, again using the values from Grupe et al. (2004). We find again that the two populations are very different, with the low $\sigma$ population having flatter spectra. The $\mathrm{K}-\mathrm{S}$ test probability of their being drawn from the same population is $P=0.2$.

It is also interesting to note that the objects with high $\sigma$ are also the ones with large FeII equivalent widths (Fig. 3). While this is not directly related to the proposed $\mathrm{BH}$ growth hypothesis, it once again shows that NLS1s is a mixed bag. Only NLS1s with steep X-ray spectra appear to be those with high $L / L_{\text {Eddington }}$ and large FeII equivalent widths (see also Grupe 2004).

All these results clearly depend upon the chosen boundary between the low and high $\sigma$ objects. The boundary at $\log \sigma_{\text {[OIII }]}=2.25$ used above divides the total NLS1 sample of 32 into two subsamples of 18 and 14 objects with low and high $\sigma$ respectively. Moving the boundary significantly either to a lower or higher value of $\sigma$ would result in less than 10 objects in one data set or the other. Nonetheless, to test the robustness of the above results we moved the $\sigma$ boundary to $\log \sigma_{\text {[OIII] }}=2.3$ which resulted in 20 objects in low $\sigma$ and 12 objects in high $\sigma$ sets. We find that the subsamples are still different with a probability of being drawn from the same population $P=0.2$ for $L / L_{\text {Eddington }}$ and $P=0.3$ for $\alpha$. Even

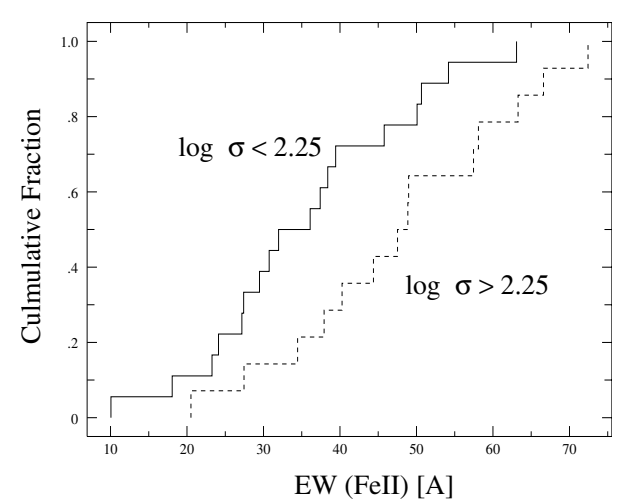

Fig. 3. Same as Fig. 1, but for equivalent width of FeII emission. Large

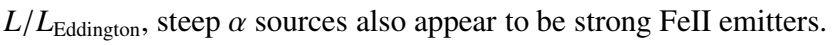

though the significance of the difference goes down away from the middle boundary, the high $\sigma$ objects always have preferentially high $L / L_{\text {Eddington }}$.

As an additional test, we also determined whether the difference $\Delta M_{\mathrm{BH}}$ between expected $\mathrm{BH}$ mass (from the $M_{\mathrm{BH}}-\sigma$ relation) and the observed mass is correlated with $L / L_{\text {Eddington. Using the Spearman rank correlation, we find that }}$ $\Delta M_{\mathrm{BH}}$ and $L / L_{\text {Eddington }}$ are correlated to better than $99.9 \%$ significance in the entire sample of 75 AGNs in Grupe \& Mathur

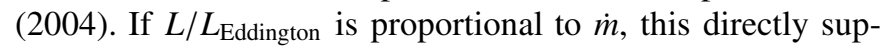
ports the hypothesis of accretion growth of black holes.

\section{Discussion}

The above tests confirm our hypothesis that NLS1s on or close to $M_{\mathrm{BH}}-\sigma$ relation have flatter $\alpha$ and emit at a lower fraction of their Eddington luminosity. We emphasize again that this result is statistical, and is robust in spite of the large errors on each of the quantities. These results have significant impact on the NLS1 paradigm which we elaborate in Williams et al. (2004); here we concentrate only on the implication for the black hole growth scenario.

The above hypothesis and tests support the scenario first presented in Mathur et al. (2001) and confirmed by Grupe \& Mathur (2004): in their high accretion phase, black holes grow substantially in mass. As they grow, they approach the $M_{\mathrm{BH}}-\sigma$ relation for normal galaxies. The mass growth in the low accretion phase, as in BLS1s and also in some NLS1s, appears to be insignificant. Any theoretical model attempting to explain the $M_{\mathrm{BH}}-\sigma$ relation needs to explain the above observations.

Needless to say, it is vital to measure $M_{\mathrm{BH}}$ and $\sigma$ accurately to critically test the above result. Black hole mass estimates based on $\mathrm{H} \beta$ widths are quite secure, but the same cannot be said about estimates of $\sigma$ based on [OIII] widths. Even if FWHM([OIII]) is not a good surrogate for $\sigma$, the nature of our result is such that $\sigma_{[\mathrm{OIII}]}-\sigma$ will have to be different for BLS1s and NLS1s, and this is most likely not the case, as discussed in Paper I. Moreover, there is no observational result to support such a difference. If NLS1s had larger outflows, then they could have disturbed their narrow-line regions more compared to BLS1s. Again, there are no observations supporting such a 
case; on the contrary, absorbing outflows are seen less often in NLS1s (Leighly 1999). Larger $L / L_{\text {Eddington }}$ in NLS1s does not necessarily imply larger effective radiation pressure. On the contrary, in objects with large soft X-ray excesses, like NLS1s, the absorbed radiation is actually much less (Morales \& Fabian 2002). There is also a general lore that highly accreting sources with large $\dot{m}$ should have large outflows. While low efficiency accretion must lead to outflows (as in ADIOS, Blandford \& Begelman 1999), the same is not true for efficient accretion as in bright Seyferts and quasars. Large outflows are observed in highly accreting sources like broad absorption line quasars (BALQSOs), but that depends upon the ratio of gas supply to Eddington accretion rate, and is not inherent to the accretion process itself (R. Blandford, private communication).

Bulge velocity dispersion is usually measured with the CaII triplet line and this technique has been used to measure $\sigma$ in two NLS1s (Ferrarese et al. 2001). However, for many of the NLS1s in our sample, the CaII lines fall in the water vapor band in the Earth's atmosphere. In many NLS1s for which CaII line is accessible from ground, CaII is observed in emission rather than in absorption (Rodriguez-Ardila et al. 2002). This makes the use of CaII absorption features to determine $\sigma$ difficult for the targets of interest. We plan to use two different methods for alternative estimates of $\sigma$ : (1) use the CO absorption band-head at 2.29 microns to measure $\sigma$ directly; and (2) use high resolution imaging of NLS1 host galaxies to measure the surface brightness distribution of bulges. One can then use fundamental plane relations to determine $\sigma$. Alternatively, we will determine the bulge luminosities and find the locus of NLS1s on the $M_{\mathrm{BH}}-L_{\mathrm{Bulge}}$ relation. Once again, the objective is to find out if there exists a statistical difference in the relation between black hole mass and bulge luminosity for the two populations of BLS1s and NLS1s. We plan to use all these methods to determine the locus of highly accreting AGNs on the $M_{\mathrm{BH}^{-}}$ bulge relations and so fully understand the role of accretion on black hole growth.

\section{References}

Adams, F. C., Graff, D. S., \& Richstone, D. O. 2001, ApJ, 551, L31 Bian, W., \& Zhao, Y. 2004, MNRAS, 347, 607
Blandford, R., \& Begelman, M. 1999, MNRAS, 303, L1

Boller, T., Brandt, W. N., \& Fink, H. H. 1996, A\&A, 305, 53

Ferrarese, L., \& Merritt, D. 2000, ApJ, 539, L9

Ferrarese, L., Pogge, R. W., Peterson, B. M., et al. 2001, ApJ, 555, L55

Gebhardt, K., Bender, R., Bower, G., et al. 2000, A\&A, 539, L13

Gebhardt, K., Kormendy, J., Ho, L. C., et al. 2000, ApJ, 543, L5

Grupe, D., Beuermann, K., Thomas, H.-C., Mannheim, K., \& Fink, H. H. 1998, A\&A, 330, 25

Grupe, D., Wills, B. J., Leighly, K. M., \& Meusinger, H. 2004, AJ, 127,156

Grupe, D. 2004, AJ, 127, 1799

Grupe, D., \& Mathur, S. 2004, ApJ, 606, L41 (Paper I)

Haehnelt, M. 2003, Classical and Quantum Gravity, 20, S31

Haehnelt, M. G., \& Kauffmann, G. 2000, MNRAS Lett., 318, 35

Haehnelt, M. G., Natarajan, P., \& Rees, M. J. 1998, MNRAS, 300, 817

Ho, L. C. 1999, in Observational Evidence for the Black Holes in the Universe, 157

King, A. 2003, ApJ, 596, L27

Kormendy, J., \& Richstone, D. 1995, ARA\&A, 33, 581

Kuraszkiewicz, J., Wilkes, B., Czerny, B., \& Mathur, S. 2000, ApJ, 542,692

Leighly, K. M. 1999, ApJS, 125, 317

Magorrian, J., Tremaine, S., Richstone, D., et al. 1998, AJ, 115, 2285

Mathur, S. 2000, MNRAS, 314, L17

Mathur, S., Kuraszkiewicz, J., \& Czerny, B. 2001, New Astron., 6, 321

Merritt, D., \& Ferrarese, L. 2001, ApJ, 547, 140

Miralda-Escudé, J., \& Kollmeier, J. A. 2004, ApJ, submitted [arXiv: astro-ph/0310717]

Morales, R., \& Fabian, A. 2002, MNRAS, 329, 209

Osterbrock, D. E., \& Pogge, R. W. 1985, ApJ, 297, 166

Pounds, K. A., Done, C., \& Osborne, J. 1995, MNRAS, 277, L5

Haering, N., \& Rix, H.-W. 2004 [arXiv:astro-ph/0402376]

Rodriguez-Ardila, A., Viegas, S. M., Pastoriza, M. G., \& Prato, L. 2002, ApJ, 565, 140

Wandel, A. 2002, ApJ, 565, 762

Wang, Y., \& Biermann, P. 1998, A\&A, 334, 87

Wang, Y., Biermann, P., \& Wandel, A. 2000, A\&A, 361, 550

Williams, R., Mathur, S., \& Pogge, R. 2004, ApJ, 610, L737

Yu, Q., \& Lu, Y. 2004 ApJ, 610, 93 